\title{
Scopolamine Reduces Persistent Activity Related to Long- Term Encoding in the Parahippocampal Gyrus during Delayed Matching in Humans
}

\author{
Karin Schon, ${ }^{1,2}$ Alireza Atri, ${ }^{2,5}$ Michael E. Hasselmo, ${ }^{1,2,3}$ Marisa D. Tricarico, ${ }^{2}$ Matthew L. LoPresti, ${ }^{2,3}$ and \\ Chantal E. Stern $1,2,3,4$ \\ ${ }^{1}$ Department of Psychology, ${ }^{2}$ Center for Memory and Brain, and ${ }^{3}$ Program in Neuroscience, Boston University, Boston, Massachusetts $02215,{ }^{4}$ Athinoula A. \\ Martinos Center for Biomedical Imaging, Massachusetts General Hospital, Charlestown, Massachusetts 02129, and ${ }^{5}$ Department of Neurology, \\ Massachusetts General Hospital and Harvard Medical School, Boston, Massachusetts 02114
}

\begin{abstract}
Recent computational modeling and slice physiology studies have suggested that long-term encoding may depend on sustained spiking during brief memory delays in parahippocampal neurons, and that this persistent spiking activity is modulated by effects of acetylcholine at muscarinic receptors. Our recent functional magnetic resonance imaging (fMRI) study has shown that sustained parahippocampal delay period activity during delayed match-to-sample performance in healthy young individuals predicted subsequent memory of visual stimuli on a recognition memory assessment 20 min later (Schon et al., 2004). The current study combined this fMRI paradigm with a pharmacological manipulation to test whether this long-term encoding-related delay activity is reduced in subjects who receive the muscarinic cholinergic antagonist scopolamine before fMRI scanning. Subsequent memory was predicted by sustained activity during brief memory delays bilaterally in the perirhinal/entorhinal cortex, in the right posterior parahippocampal and mid-fusiform gyri, and in the hippocampal body in healthy young individuals without a scopolamine challenge. This activity was reduced in subjects receiving scopolamine. The results are consistent with computational modeling data and behavioral pharmacological studies, suggesting that long-term encoding-related activity may be reduced if cholinergic receptors are blocked by scopolamine.
\end{abstract}

Key words: memory formation; cholinergic; fMRI; medial temporal lobe; delayed match to sample; computational modeling

\section{Introduction}

Recent computational models have shown that maintenance of information in working memory for long-term encoding may depend on cholinergic activation of intrinsic mechanisms for sustained spiking activity (Lisman and Idiart, 1995; Jensen and Lisman, 1996; Fransén et al., 2002; Hasselmo et al., 2002b; Koene et al., 2003). Data from slice preparations have shown that muscarinic cholinergic receptors activate an intrinsic cation current that causes sustained spiking activity in neurons of entorhinal cortex (Klink and Alonso, 1997; Egorov et al., 2002). Computational models that incorporate this current can simulate spiking activity during a delayed match-to-sample (DMS) task (Fransén et al., 2002). In a typical DMS task, the subject is instructed to remember the sample stimulus over a brief memory delay. When the test stimulus appears at the end of the delay, the subject has to

Received May 17, 2005; revised Aug. 9, 2005; accepted Aug. 20, 2005

This work was supported by National Institutes of Health (NIH) Grants ROI NS41636 (C.E.S.) and ROI MH61492, MH60013, and DA16454 (M.E.H.), as part of the NIH/National Science Foundation Collaborative Research in Computational Neuroscience program, and by a Boston University Clara Mayo Fellowship (K.S.). We thank all of our volunteers who participated in this study and two anonymous reviewers for their helpful comments and suggestions on a previous version of this manuscript. Parts of this paper have been presented previously at the 34th Annua Meeting of the Society for Neuroscience, San Diego, CA (2004).

Correspondence should be addressed to Dr. Chantal E. Stern, Center for Memory and Brain, Boston University, 2 Cummington Street, Boston, MA 02215. E-mail: chantal@bu.edu.

DOI:10.1523/JNEUROSCI.1982-05.2005

Copyright $\odot 2005$ Society for Neuroscience $\quad$ 0270-6474/05/259112-12\$15.00/0 indicate whether or not the test stimulus matches the sample stimulus. Sustained neuronal activity during brief memory delays has been observed in parahippocampal neurons of monkeys performing delayed matching tasks (Suzuki et al., 1997; Young et al., 1997). Fransén et al. (2002) showed that this sustained spiking during memory delays could result from high levels of acetylcholine, which activate the intrinsic current, whereas lower levels of cholinergic activation would result in an absence of persistent spiking activity.

The cholinergic enhancement of persistent spiking activity may contribute to long-term encoding, in particular if novel stimuli are being encoded, consistent with previous functional magnetic resonance imaging (fMRI) studies examining the role of the medial temporal lobe (MTL) in working memory (Ranganath and D'Esposito, 2001; Stern et al., 2001). Previous studies indicate a role for acetylcholine in encoding. Behavioral studies in monkeys (Aigner et al., 1991; Tang et al., 1997) and humans (Ghoneim and Mewaldt, 1975, 1977; Rosier et al., 1998; Atri et al., 2004) have shown that injection of the muscarinic cholinergic antagonist scopolamine before encoding, but not before retrieval, impairs performance on memory tasks. A recent fMRI study showed that scopolamine reduces hippocampal, fusiform, and inferior prefrontal activity in individuals performing a longterm associative encoding task (Sperling et al., 2002). Similarly, a positron emission tomography study demonstrated that the in- 
fluence of scopolamine at encoding reduces recognition-related activity in the fusiform gyrus (FG) (Rosier et al., 1999).

Based on the computational modeling and experimental data described above, we predicted that blockade of acetylcholine receptor activation should reduce sustained activity associated with encoding. This prediction was tested by combining a cholinergic challenge with the paradigm from our previous fMRI study, which demonstrated that activation during the delay period of a DMS task was correlated with subsequent performance on a recognition memory task (Schon et al., 2004). We hypothesize that scopolamine injection before the DMS task should reduce activity in the parahippocampal gyrus (PHG) during brief memory delays that predicts subsequent memory.

\section{Materials and Methods \\ Subjects}

Thirty-one healthy young individuals participated in this study. Subjects were recruited from the Boston University community. Fifteen subjects received scopolamine before encoding (SCOP group; for details, see below, Pharmacological procedures). Sixteen additional subjects did not receive any drug injection (NO DRUG group). Data from the NO DRUG group has been reported in our study examining delay period activity during the DMS task (Schon et al., 2004). This group served as the control group for this study. The SCOP group and the NO DRUG group were group matched by age (NO DRUG, $22.9 \pm 4.7$; SCOP, $22.1 \pm 3.6$; $\left.t_{(29)}=0.4933 ; p_{2 \text {-tailed }}=0.6255\right)$. In each group, 10 participants were female. Vision was normal or corrected-to-normal. All subjects were screened for history of psychiatric or neurologic symptoms. Before injection with scopolamine, the subjects underwent a comprehensive medical examination, including a pregnancy test for female subjects, and were not included if they had a history of any significant medical condition that could be aggravated by anticholinergic drugs or if they took any neuropsychiatric drugs. All subjects signed informed consent in a manner approved by both the Human Research Committee of the Massachusetts General Hospital and the Boston University Institutional Review Board and received nominal monetary compensation for participating in this study.

\section{Pharmacological procedures}

Subjects in the SCOP group received an intramuscular injection of 0.4 $\mathrm{mg}$ of scopolamine in a $1 \mathrm{ml}$ saline solution $\sim 90$ min before the beginning of the functional scans. Scopolamine is a medication that has been used in a variety of clinical applications, including treatment of gastrointestinal disorders, as a surgical preanesthetic, and as treatment for motion sickness. Scopolamine, at this dosage and higher, has been used in studies to assess the effects of cholinergic blockade on human memory function (Ghoneim and Mewaldt, 1975, 1977; Sherman et al., 2003; Atri et al., 2004). We selected this relatively low dose to avoid sedation. The subjects were periodically reexamined, their vital signs, including pulse, blood pressure, and respiratory rate, were recorded, and they were monitored for potential side effects. The subjects were observed for $4 \mathrm{~h}$ after injection and until the study physician determined that the side effects of the drug had subsided.

As expected effects of scopolamine at this dosage, subjects who received the drug experienced transient pupillary dilation, mild-tomoderate dryness of the mouth, and a subjective perception of being mildly altered by a drug. Of these subjects, nine felt mildly to moderately tired, and five felt mildly lightheaded for a short time. There were no significant changes in pulse rate, blood pressure, respiratory rate, or neurological exam.

\section{Behavioral procedures}

DMS task. To assess sustained activity during brief memory delays, we used a DMS task with digitized photographs of trial-unique complex visual scenes as stimuli, and a control (CON) task that differed from the DMS task only in instruction and scoring (Fig. 1A) (Schon et al., 2004). In both tasks, each trial consisted of a $2 \mathrm{~s}$ visual scene presentation (sample), followed by a $10 \mathrm{~s}$ delay period, followed by a $2 \mathrm{~s}$ visual scene

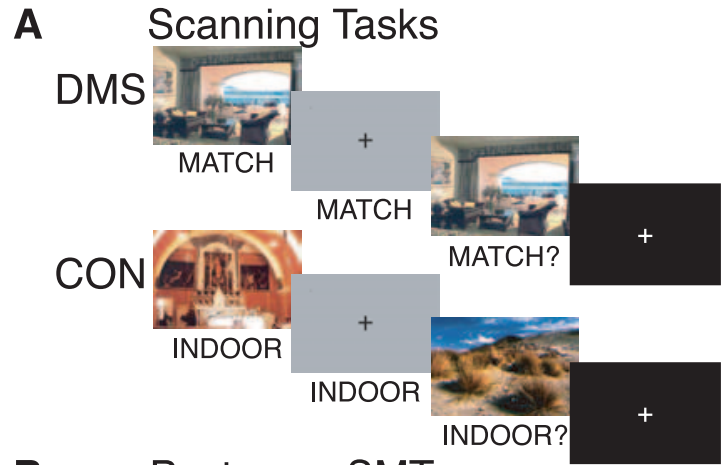

B

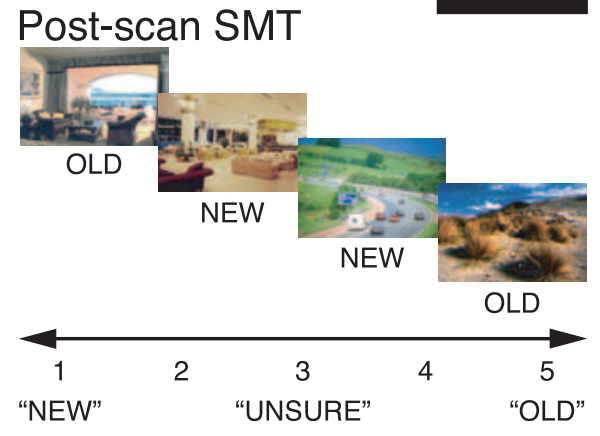

Figure 1. Behavioral tasks. $\boldsymbol{A}$, Scanning tasks. Subjects saw the sample picture for $2 \mathrm{~s}$, followed by a 10 s delay during which a gray box appeared, followed by a test picture presentation for $2 \mathrm{~s}$, followed by a variable-length ITI (6-14 s) during which a fixation cross appeared. DMS and $\mathrm{CON}$ tasks differed only in instruction. The DMS task required the subject to indicate whether the test picture matched the sample picture, and the $\mathrm{CON}$ task required the subject to indicate whether the test picture was indoors or outdoors. B, Post-scan SMT. The subject saw all stimuli again from both DMS and CON tasks and an equal number of new stimuli. The task required the subject to indicate on a five-point rating scale their confidence on whether a given picture was old (previously seen) or new (never seen before). Stimuli were shown on black background.

presentation (test), followed by a variable-length intertrial interval (ITI) $(6,10$, or $14 \mathrm{~s})$. The variable ITI introduces temporal jitter for selective averaging and provides time for the hemodynamic response of the previous trial to return to baseline (Postle et al., 2000). A visual cue presented during sample, delay period, and test presentations instructed the subject. The cue "MATCH" instructed the subject to remember the sample picture during the delay period. During the test period, the cue "MATCH?" prompted the subject to make a yes/no response on whether or not the test picture matched the sample picture by using a button box (DMS task). Half of the trials were match trials ("yes" responses), and half were nonmatch trials ("no" responses). The cue "INDOOR" prompted the subject to simply wait until the test picture appeared. During the test period, the cue "INDOOR?" prompted the subject to indicate whether or not the test picture was indoors (CON task). No reference back to the sample picture was necessary to perform this task accurately. These tasks were designed such that memory was maximized during the DMS delay period and minimized during the CON delay period, while keeping visuomotor requirements constant. There were 12 parallel sets of eight runs, and each run always included six DMS and six CON trials. Although each subject performed eight runs during fMRI scanning and each run always had six DMS trials and six CON trials, the order of conditions and stimuli used was not identical. The 12 sets differed with regard to condition order and with regard to stimulus selection. Conditions were counterbalanced in each of the 12 sets, and stimulus selection was always random within a given category (indoor scenes vs outdoor scenes). Before scanning, subjects viewed task instructions on a computer screen and practiced the tasks. Stimuli were presented, and reaction times (RTs) and errors were recorded with PsyScope 1.2.5 (Cohen et al., 1993).

Post-scan subsequent memory assessment. Approximately $20 \mathrm{~min}$ after fMRI scanning, subjects performed a surprise self-paced subsequent rec- 
ognition memory task (SMT) (Fig. 1B) (Schon et al., 2004). Subjects viewed all stimuli presented as sample and test stimuli during both DMS and CON trials and an equal number of new pictures (lures) in random order. They were instructed to rate on a five-point scale their confidence as to whether or not a displayed scene had been presented previously during the DMS and CON tasks. The rating scale (Fig. $1 B$ ) ranged from 1 to 5 (1, high confidence new; 2, low confidence new; 3 , unsure; 4 , low confidence old; and 5, high confidence old) (Fig. $1 B$ ). We have used this scale previously in our laboratory to assess subsequent recognition (Sherman et al., 2003; Schon et al., 2004), and these behavioral procedures are identical to those described by Schon et al. (2004).

Statistical analysis. We used repeated-measures ANOVAs to assess the effects of group (NO DRUG vs SCOP) and task (DMS vs CON) on RTs and proportion of correct responses. Because RTs follow a skewed, nonGaussian distribution, we first calculated median RTs for individual subjects and then calculated group means based on the medians.

The response proportions on the post-scan SMT were analyzed statistically with the generalized linear model (GLM) using the Huber/White/ sandwich estimator of variance to obtain robust variance estimates, and dependent variables (response proportions for DMS samples from nonmatch trials, for DMS samples from match trials, and for new stimuli) were classified as binomially distributed data. The GLM included the factors group (NO DRUG vs SCOP) and response distribution (postscan SMT scores, 1 vs 2 vs 3 vs 4 vs 5). This statistical procedure has been proposed for use with proportions that have a non-Gaussian distribution (Papke and Wooldridge, 1996).

Focal $t$ tests with Bonferroni-corrected $p$ values ( $p=0.05$ divided by $5=0.01$ ) were also used to compare the SMT5 scores for DMS samples (hits) and for new stimuli (false alarms) of both groups.

\section{fMRI procedures}

Data acquisition. fMRI data acquisition included collection of two highresolution T1-weighted structural images (magnetization-prepared rapid-acquisition gradient echo; field of view, $256 \times 256)$ and eight functional $\mathrm{T} 22^{\star}$-weighted gradient-echo echo planar blood oxygenation level-dependent scans (time to repetition, $2 \mathrm{~s}$; time to echo, $30 \mathrm{~ms}$; flip angle, $90^{\circ}$; field of view, $64 \times 64 ; 21$ slices per image; 149 images per scan; in-plane resolution, $3.1 \times 3.1 \mathrm{~mm}$; slice thickness, $5 \mathrm{~mm}$; skip between slices, $1 \mathrm{~mm}$ ). The data were acquired on a 3.0 tesla Siemens (Erlangen, Germany) Allegra scanner at the Athinoula A. Martinos Center for Biomedical Imaging (Massachusetts General Hospital, Charlestown, MA).

Data preprocessing. Functional scans were temporally smoothed by correcting for differences in slice timing, realigned to the first image within a series to correct for motion artifacts, unwarped to correct for movement-by-susceptibility interactions, normalized into standard Montreal Neurological Institute (MNI) space with standard resampling of $2 \times 2 \times 2 \mathrm{~mm}$ isotrophic voxels, and spatially smoothed with a $6 \mathrm{~mm}$ full-width half-maximum Gaussian kernel using SPM2 software (Wellcome Department of Cognitive Neurology, London, UK).

Statistical analysis. We investigated delay period activity in two ways: (1) we assessed active maintenance of novel information during brief memory delays regardless of later long-term memory for this information (active maintenance analysis), and (2) we assessed long-term encoding by selectively averaging the delay activity according to whether the immediately preceding sample was remembered with high confidence as assessed by the post-scan SMT (long-term encoding analysis).

Because of the time-locked nature of the events within a trial (sample period, followed by delay period, followed by test period), we used multiple regression analysis instead of conventional event-related analysis procedures (Dale and Buckner, 1997; Buckner, 1998). The delay length was kept fixed, because long and short memory delays have been shown to result in qualitatively differential activation patterns, in particular within the medial temporal lobes, even if they differ only by a few seconds (Elliott and Dolan, 1999). With multiple regression analysis, hemodynamic changes attributable to different trial components that are timelocked can be assessed simultaneously and independently (Zarahn et al., 1997; Courtney et al., 1998; Postle et al., 2000). This approach has been used previously (Courtney et al., 1997, 1998; Postle and D'Esposito, 1999a,b; Ranganath and D'Esposito, 2001; Schon et al., 2004). For the active maintenance analysis, we modeled the regressors following Courtney et al. (1998). We used the following six contrasts. The first contrast assessed activity during visual stimulation (sample and test stimuli, across tasks) versus activity during the absence of visual stimulation (delay periods and ITIs, across tasks). The second contrast assessed activity attributable to DMS stimuli (samples and tests) versus activity attributable to CON stimuli (samples and tests). The third contrast assessed activity during the CON sample period versus activity during the CON test period and motor response. The fourth assessed the same as the third for DMS stimuli. The fifth assessed activity during delay periods across tasks versus activity during ITIs. The sixth contrasted activity during the DMS delay versus activity during the CON delay. We convolved the six contrasts with a gamma-variate function (Boynton et al., 1996). The convolved contrasts constituted the six regressors. Including all six regressors simultaneously into the multiple regression analysis allowed us to isolate activity that was solely attributable to the memory delay of the task (regressor 6), because the other regressors accounted for all other task components (regressors 1-5) (Courtney et al., 1998). Here we report results from regressor 6.

For the long-term encoding analysis, we used the following eight contrasts. The first contrast assessed activity during sample periods from DMS match trials of sample stimuli that were subsequently remembered with high confidence on the post-scan SMT (SMT5) versus those that were not (SMT1-4). The second contrast assessed the same as the first for sample periods from DMS nonmatch trials. The third contrast assessed activity during delay periods of DMS match trials after sample stimuli that were remembered with high confidence on the post-scan SMT (SMT5) versus all other ratings (SMT1-4). The fourth contrast assessed the same as the third contrast for delay periods of nonmatch trials. The fifth contrast assessed activity during sample presentation periods from CON trials for sample stimuli that were subsequently remembered with high confidence on the post-scan SMT (SMT5) versus all other ratings (SMT1-4). The sixth contrast assessed activity during delay periods from CON trials after sample stimuli that were remembered with high confidence on the post-scan SMT (SMT5) versus all other ratings (SMT1-4). The seventh contrast assessed test presentation periods from DMS trials of test stimuli that were subsequently remembered with high confidence on the post-scan SMT (SMT5) versus all others (SMT1-4). The eighth contrast assessed the same as the seventh for test presentation periods from CON trials. We convolved the eight contrasts with a gamma-variate function (Boynton et al., 1996). The convolved contrasts constituted the eight regressors. This multiple regression analysis with eight regressors allowed us to isolate activity that was solely attributable to sustained encoding during the DMS task delay separately for match trials (regressor 3 ) and for nonmatch trials (regressor 4), and that was independent from other task components, because these were accounted for by all other regressors (regressors 1,2, and 5-8). Here we report results for regressors 3 and 4 . Match and nonmatch trials were analyzed separately because, in the case of match trials, the sample picture was seen twice, once during sample and once during test presentation, and, in the case of nonmatch trials, the sample pictures were seen only once during sample presentation before the subjects saw them again on the post-scan SMT. Because it was more likely that a sample picture was remembered later with high confidence when it was previously seen twice (during match trials) than when it was seen once (during nonmatch trials), we analyzed match and nonmatch trials separately. We compared SMT scores of 5 (SMT5) with SMT scores of 1-4 (SMT1-4), because we were interested in recollection-based, not familiarity-based, behavioral responses on the post-scan SMT (Yonelinas, 1994; Sherman et al., 2003; Schon et al., 2004). To increase statistical power, we performed a secondary analysis that collapsed across match and nonmatch trials (i.e., regressors 1 and 2, as well as 3 and 4, were combined).

For both analyses and for each subject, the regressors were entered into SPM2 for statistical analysis. The regressors were created such that they were orthogonal or nearly orthogonal to each other. This allowed us to interpret the results independently for each regressor. Random-effects group analyses with subjects as the random factor were performed on each regressor by entering the resulting $t$-contrast image of each subject into second-level one- and two-sample $t$ tests. 
For the fMRI result assessment of the active maintenance analysis, we used a statistical threshold of $p<0.05$, small-volume corrected (Worsley et al., 1996) using the false discovery rate (FDR) procedure (Genovese et al., 2002), in conjunction with a threshold extent of five voxels. This small-volume correction was performed based on a large structurally defined region-of-interest (ROI) mask (see below). Because we focus the subsequent memory analyses on DMS trials, we reported activity as statistically significant at $p_{\text {uncor }} \leq 0.001$ (uncorrected for multiple voxelwise comparisons) with a threshold extent of five voxels for this analysis.

ROI definition. Our temporal lobe ROI was composed of the PHG [including perirhinal and entorhinal cortex (PRC/ERC)], the hippocampus, and the FG, bilaterally, because previous fMRI studies of long-term encoding have demonstrated subsequent memory effects in these regions (Brewer et al., 1998; Fernàndez et al., 1998, 1999; Wagner et al., 1998; Kirchhoff et al., 2000; Davachi and Wagner, 2002; Davachi et al., 2003). We defined these regions structurally using the WFU_PickAtlas tool (Maldjian et al., 2003), which allows automated atlas-based masking in conjunction with the SPM software for assessment of statistical results restricted to a priori defined ROIs. We used the International Consortium for Brain Mapping (ICBM) atlas to structurally define our ROI (Mazziotta et al., 2001) in MNI space.

Time series extraction and analysis. We extracted average signal intensities for each time point from a $5 \mathrm{~mm}$ sphere around functional peak activations within our temporal lobe ROI adjusted for all effects of interest using the VOI tool of the SPM2 software. Unless stated otherwise, the coordinates of the functional peak activations were taken from the onesample $t$ test group analysis of the NO DRUG group, because activity in the NO DRUG group provided the baseline for comparison of the SCOP group data. Alternatively, it has been suggested to use functional peak coordinates from a composite map based on all subjects such that one group is not favored over another for time series extraction (Buckner et al., 2000). In an additional analysis, signal intensities were extracted from coordinates of the functional peak activations from a one-sample $t$ test of all subjects combined (NO DRUG and SCOP). Data from this analysis are provided as supplemental data (available at www.jneurosci.org as supplemental material). We selectively averaged the time series by group (NO DRUG vs SCOP), task (DMS vs CON), memory (SMT5 vs SMT14), and event (SAMPLE vs DELAY vs TEST) for the active maintenance analysis, and by group (NO DRUG vs SCOP), DMS trial (MATCH vs NONMATCH), memory (SMT5 vs SMT1-4), and event (SAMPLE vs DELAY vs TEST) for the long-term encoding analysis. Repeated-measures ANOVAs assessed main effects and interactions of these factors. We used a statistical threshold of $p=0.05$ (GeisserGreenhouse corrected, when applicable). Repeated-measures ANOVAs with these within-subject factors used the four-way interaction as the between-subject error term.

\section{Results}

The main finding of this paper is that delay period activity in the anterior parahippocampal gyrus that predicted subsequent memory in healthy young individuals (NO DRUG group) is attenuated in subjects who received the muscarinic receptor blocker scopolamine (SCOP group). This result is described below (see Long-term encoding-related activity during the DMS delay). Scopolamine also reduced active maintenance-related activity in mid-fusiform/parahippocampal regions, but this effect was not as strong as the subsequent memory effect (see below, Active maintenance of novel information). In addition, we also observed behavioral changes in the response distributions on the SMT, with a reduced number of high confidence hits (SMT5) in the SCOP group (see below, Behavioral performance).

\section{Behavioral performance}

\section{Performance on DMS and CON tasks}

A repeated-measures ANOVA with group (NO DRUG vs SCOP) as the between-subjects factor and task (DMS vs CON) as the repeated measure on median RTs revealed a main effect of group
A

\section{DMS and CON Tasks}
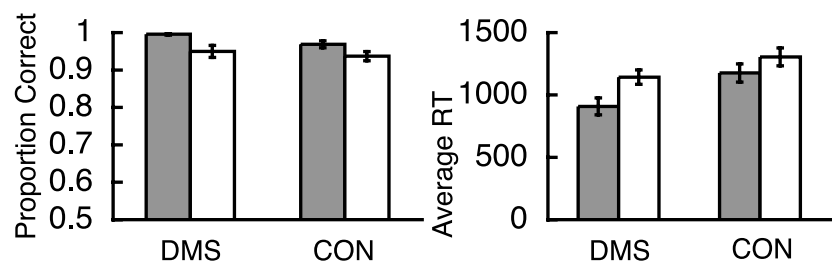

\section{B Post-scan SMT}

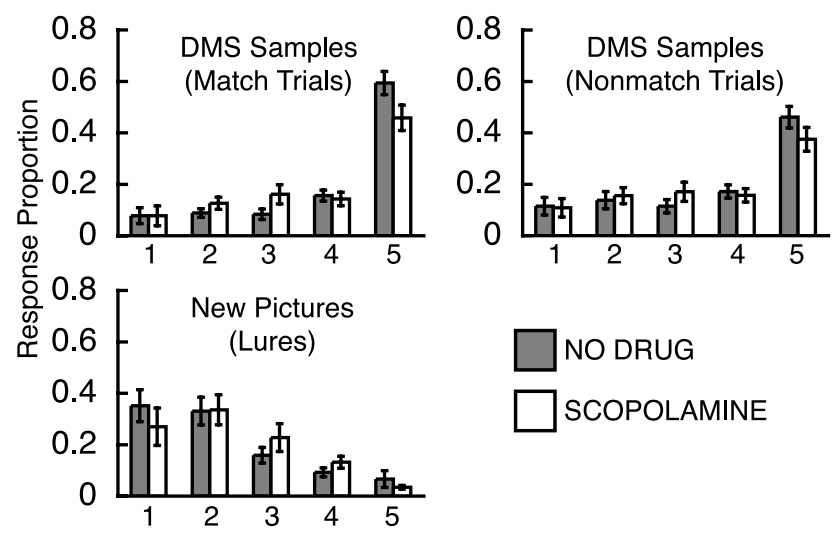

Figure 2. Behavioral results. $\boldsymbol{A}$, The proportions of correct responses on DMS and CON task performance are depicted on the left, and the mean of the median RTs (in milliseconds) on DMS and CON task performance are depicted on the right. $\boldsymbol{B}$, Response proportions on postscan subsequent memory rating for sample stimuli that were seen twice (match trials; top left), for sample stimuli that were seen once (nonmatch trials; top right), and for new stimuli (lures; bottom left) that were not seen before the postscan SMT was performed. The rating scale ranged from 1 (high confidence new) to 5 (high confidence old). Gray bars depict results for the NO DRUG group, and white bars depict results for the SCOP group.

$\left(F_{(1,56)}=7.17 ; p=0.0097\right)$ and a main effect of task $\left(F_{(1,56)}=\right.$ $10.06 ; p=0.0025)$, indicating that, on average, the SCOP group was slower than the NO DRUG group on both tasks [mean RTs \pm SD (in milliseconds); DMS, $1143 \pm 224$ vs $908 \pm 263$, respectively; CON, $1305 \pm 278$ vs $1176 \pm 283$, respectively], and that both groups were slower on the CON task compared with the DMS task (Fig. 2A). Each group performed both tasks at high levels ( $\geq 94 \%$ correct responses). A repeated-measures ANOVA on the proportion of correct responses also revealed a main effect of group $\left(F_{(1,56)}=12.05 ; p=0.001\right)$, indicating that the SCOP group made more errors on both tasks in the scanner compared with the NO DRUG group (DMS, $0.950 \pm 0.062$ vs $0.996 \pm$ 0.009 , respectively; CON, $0.937 \pm 0.047$ vs $0.969 \pm 0.036$, respectively). The significant difference between the two groups is most likely attributable to the ceiling performance of the NO DRUG group on the DMS task that resulted in reduced variance (Fig. $2 A)$. In addition, there was a statistical trend toward a main effect of task $\left(F_{(1,56)}=3.12 ; p=0.0826\right)$, indicating that, overall, both groups made slightly more errors on the DMS task than on the CON task.

\section{Performance on the post-scan SMT}

Overall, the SCOP group recognized slightly fewer sample pictures with high confidence than the NO DRUG group, but otherwise both groups had similar scores on the post-scan recognition memory assessment (SMT).

The GLM analysis on the response proportions of sample pictures that were encountered once (nonmatch trials) and those of sample pictures that were encountered twice (match trials) before 
the post-scan SMT revealed a significant main effect of the response proportions $\left(Z_{(157)}=7.37, p<0.001\right.$; and $Z_{(157)}=7.45$, $p<0.001$, respectively); however, on overall recognition, the two groups did not differ significantly $\left(Z_{(157)}=-1.00\right.$, $p=0.315$; and $Z_{(157)}=-1.00, p=0.315$, respectively) (Fig. $2 B$ ). A focal one-tailed $t$ test on response proportions of sample pictures revealed a trend showing a slightly greater proportion of hits (SMT5) for the NO DRUG group compared with the SCOP group (sample pictures seen twice, $t_{(29.912)}=2.2323, p_{1 \text {-tailed }}=0.0166$; sample pictures seen once, $t_{(29.6099)}=$ $\left.1.6531, p_{1 \text {-tailed }}=0.0544\right)$ (Fig. 2 B), indicating that the SCOP group recognized slightly fewer DMS sample pictures with high confidence on the post-scan SMT (SMT5) (Fig. 2B). The GLM analysis on the response proportions to new stimuli (lures) revealed both a significant main effect of response distribution $\left(Z_{(157)}=\right.$ $-5.63 ; p<0.001)$ and a trend toward an effect of group $\left(Z_{(157)}=-1.79 ; p=\right.$ $0.073)$, indicating that the NO DRUG and SCOP groups differed slightly but not significantly in their old/new confidence ratings on the post-scan SMT (Fig. 2 B). The two groups did not differ on the proportion of false alarms (SMT5), as indicated by a nonsignificant two-tailed $t$ test $\left(t_{(16.4291)}=0.8552 ; p_{2 \text {-tailed }}=0.2024\right)$. Consistent with this result, discriminability $d^{\prime}\left(Z_{\text {false alarms }}-Z_{\text {hits }}\right)$ did not differ between the two groups $\left(t_{(48.4834)}=\right.$ $\left.1.4836 ; p_{2 \text {-tailed }}=0.1444\right)$, nor did response bias $c\left[\left(Z_{\text {false alarms }}+\right.\right.$ $\left.\left.Z_{\text {hits }}\right) / 2 ; t_{(52.91)}=-1.3411 ; p_{2 \text {-tailed }}=0.1856\right]$.

\section{fMRI results}

\section{Active maintenance of novel information}

The active maintenance analysis on the NO DRUG data revealed that, when activity during the DMS delay was contrasted with activity during the CON delay, regardless of long-term subsequent memory, there were two activation foci within our ROI (Fig. 3A), one in the right mid-FG/PHG (peak at $x=34, y=-34$, $\left.z=-20 ; Z=4.18 ; p_{\mathrm{FDR}}=0.02\right)$ and one in the left mid-FG/PHG (peak at $x=-32, y=-48, z=-12 ; Z=3.60 ; p_{\mathrm{FDR}}=0.02$ ). When the same analysis was done on the SCOP data, the activation in the right mid-FG/PHG was not present, and the activation in the left mid-FG/PHG was reduced in both magnitude and extent (peak at $x=-28, y=-38, z=-22 ; Z=2.62 ; p_{\text {uncor }}=$ 0.004 ) (Fig. $3 B$ ). Results from a two-sample $t$ test comparing the NO DRUG group with the SCOP group confirmed this finding. The NO DRUG group had significantly greater activation in the right mid-FG/PHG (peak at $x=20, y=-34, z=-18 ; Z=3.36$; $p_{\text {uncor }}<0.001$ ) (Fig. $3 C$ ) and in the left mid-FG/PHG (peak at $x=-24, y=-46, z=-18 ; Z=2.90 ; p_{\text {uncor }}=0.002$; statistical trend) (Fig. 3C).

Repeated-measures ANOVAs on extracted signal-intensity time series from the two activation peaks derived from the NO DRUG group examined the effects of group (NO DRUG vs SCOP), task (DMS vs CON), memory (SMT5 vs SMT1-4), and

\section{Right Left mid-FG/PHG mid-FG/PHG NO DRUG}
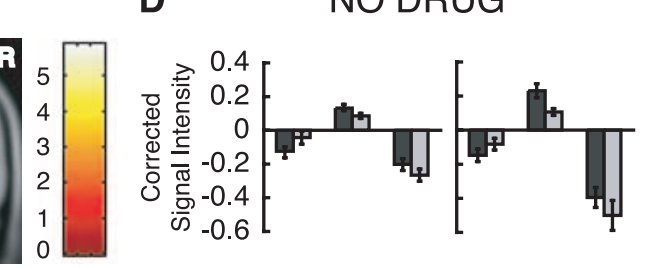

E SCOP
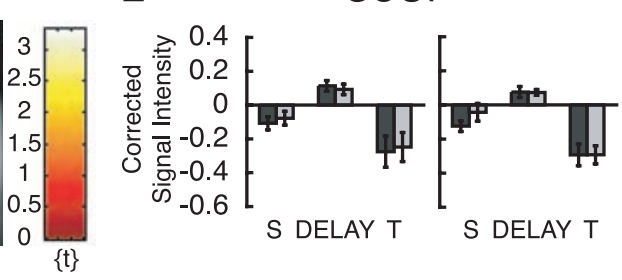

$\{\mathrm{t}\}$

$S$ DELAY T

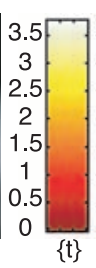

$\square$ DMS

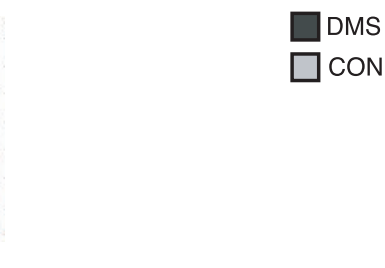

Figure 3. $\quad \mathrm{fMRI}$ activation from the active maintenance analysis. Only activation within ROls is superimposed on canonical average T1-weighted ICBM/MNI brain. $\boldsymbol{A}$, NO DRUG group shows right mid-FG/PHG activity, $y=-34$, depicted on left, and left S PHG, $y=-48$, depicted on right. $B$, Same as in A for SCOP group. C, Greater activation for the NO DRUG group compared right. $\boldsymbol{E}$, Same as $\boldsymbol{D}$ but for the SCOP group. $y$-Axes, Signal intensity grand mean scaled to 100 and global calculation using mean voxel value (within per image full mean/8 mask). R, Right hemisphere; L, left hemisphere.

event (SAMPLE vs DELAY vs TEST). Because we were interested in whether active maintenance (DMS delay activity $>$ CON delay activity) was modulated by scopolamine, we were mainly interested in finding main effects and/or interactions involving the factors task (DMS vs CON) and group (NO DRUG vs SCOP). Results are summarized in Table 1. The ANOVA on the time series from the right mid-FG/PHG revealed a significant main effect of memory $\left(F_{(1,345)}=16.43 ; p<0.001\right)$, indicating that activity in this region was greater for items that were subsequently remembered with high confidence than those that were not (SMT5 > SMT1-4; data not shown). In addition, there was a trend toward a main effect of task $\left(F_{(1,345)}=2.75 ; p=0.098\right)$, indicating that activity in the right mid-FG/PHG tended to be greater for the DMS task than for the CON task (Fig. 3D,E, graphs on left). The same repeated-measures ANOVAs performed separately for each group confirmed that this trend was attributable to a main effect of task in the NO DRUG group $\left(F_{(1,180)}=4.82 ; p=0.029\right)$ and is absent in the SCOP group $\left(F_{(1,165)}=0.09 ; p=0.761\right)$. In addition, these same separate ANOVAs illustrate that the main effect of memory was primarily driven by the NO DRUG group $\left(F_{(1,180)}=17.00, p<0.001\right.$; and $F_{(1,165)}=3.23, p=0.074$ for NO DRUG and SCOP groups, respectively). There was no significant main effect of group or significant interactions involving the factors group and task. The ANOVA on the time series from the left mid-FG/PHG peak revealed a significant group $\times$ event interaction $\left(F_{(2,345)}=4.55\right.$; $p=0.016)$ and a trend toward a group $\times$ task $\times$ memory $\times$ event 
Table 1. Repeated-measures ANOVA: active maintenance analysis, DMS delay versus CON delay

\begin{tabular}{|c|c|c|c|}
\hline \multirow[b]{2}{*}{ Source } & \multicolumn{3}{|l|}{$F$} \\
\hline & $\mathrm{df}$ & $\begin{array}{l}\text { Left Mid-FG/PHG } \\
(-32,-48,-12)^{a}\end{array}$ & $\begin{array}{l}\text { Right Mid-FG/PHG } \\
(34,-34,-20)^{a}\end{array}$ \\
\hline Group & 1 & 2.54 & 2.22 \\
\hline Task & 1 & 1.28 & $2.75^{b}$ \\
\hline Memory & 1 & 2.15 & $16.43^{* * *}$ \\
\hline Event & 2 & $69.09 * * *$ & $33.98^{* * *}$ \\
\hline Group $\times$ task & 1 & 1.91 & 1.41 \\
\hline Group $\times$ memory & 1 & 2.47 & 1.67 \\
\hline Group $\times$ event & 2 & $4.55^{*}$ & 0.24 \\
\hline Task $\times$ memory & 1 & 0.58 & 0.57 \\
\hline Task $\times$ event & 2 & 1.13 & 0.45 \\
\hline Memory $\times$ event & 2 & 0.27 & 0.11 \\
\hline Group $\times$ task $\times$ memory & 1 & 0.11 & 0.02 \\
\hline Group $\times$ task $\times$ event & 2 & 1.27 & 0.47 \\
\hline Group $\times$ memory $\times$ event & 2 & 0.02 & 0.11 \\
\hline Task $\times$ memory $\times$ event & 2 & 0.08 & 0.01 \\
\hline Group $\times$ task $\times$ memory $\times$ event & 2 & $2.38^{b}$ & 0.27 \\
\hline Errors & 345 & $(0.11)$ & $(0.12)$ \\
\hline
\end{tabular}

Note that values enclosed in parentheses represent mean square errors. ${ }^{*} p<0.05 ;{ }^{* * *} p<0.001$.

${ }^{a}$ Peak Talairach coordinates $(x, y, z)$ in MNI space.

${ }^{b}$ Statistical trend $(p \leq 0.1)$

\section{Right Right + left posterior PHG PRC/ERC}

A

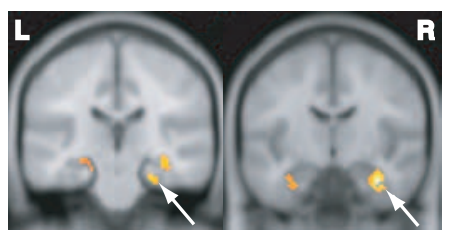

B

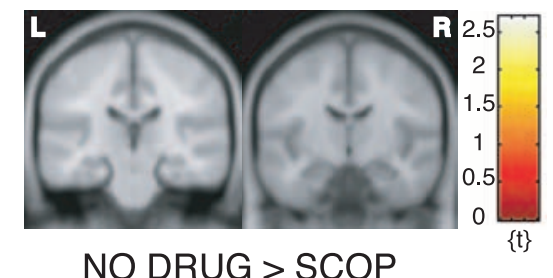

C

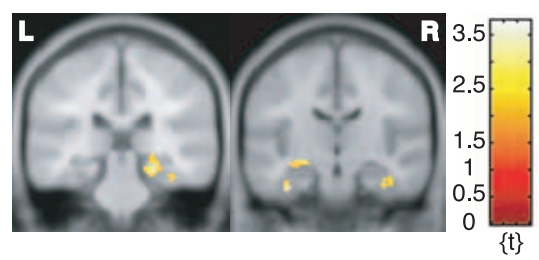

\section{Right Right posterior PHG PRC/ERC NO DRUG}

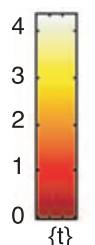

\{t\}

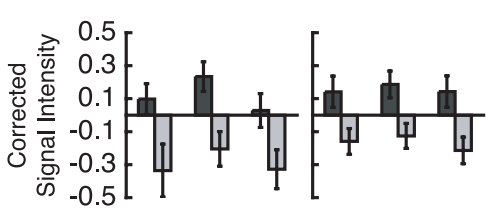

E

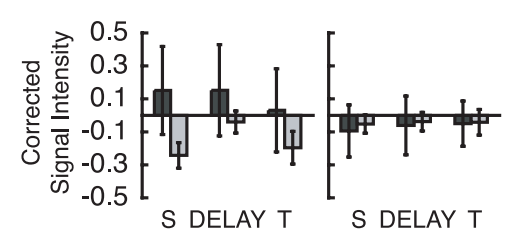

DRUG group (linear test, $F_{(1,357)}=3.08$; $p=0.080$; statistical trend) but not for the SCOP group (linear test, $F_{(1,357)}=0.02$; $p=0.899)$.

In summary, the mid-FG/PHG region, bilaterally, showed greater activity during the DMS task compared with the CON task in the NO DRUG group, but this effect was small. In addition, this active maintenance-related delay activity was slightly reduced in the SCOP group compared with the NO DRUG group.

Please note that ANOVAs were performed on signal intensities extracted from time series using coordinates from the NO DRUG group. We believe that the appropriate comparison is to use the healthy or control group as the baseline to which other groups are compared. However, we also repeated the analyses by extracting peak coordinates from a single composite statistical parametric map that was derived by entering all subjects from the NO DRUG and SCOP groups into a one-sample $t$ test (Buckner et al., 2000). Results of this analysis are described as supplemental data (available at www. jneurosci.org as supplemental material).

Long-term encoding-related activity during the DMS delay

The long-term encoding analysis showed a significant effect of the scopolamine manipulation on functional activation during delay periods correlated with performance on the post-scan SMT.

Long-term encoding analysis of the NO DRUG data examined differences in delay activity correlated with performance on the post-scan SMT. Activity during DMS delays following samples that were remembered with high confidence on the post-scan SMT (SMT5) was contrasted with activity during DMS delays following samples that were not remembered with high confidence on the SMT (SMT1-4). There were five activation foci within our ROI, when the analysis was restricted to nonmatch trials only (Figs. $4 A$, $5 A$, brain image on left), i.e., when sample stimuli were encountered only once before recognition assessment on the post-scan SMT.

These activation foci were in the right posterior PHG/mid-FG (peak at $x=24$, $y=-24, z=-24 ; Z=2.63 ; p_{\text {uncor }}=$ 0.004 ; statistical trend) (Fig. $4 A$, brain image on left), in the right PRC/ERC (peak at $x=34, y=-10, z=-28 ; Z=3.45$; $p_{\text {uncor }}<0.001$ ) (Fig. 4A, brain image on right), and left PRC/ERC (peak at $x=$ interaction $\left(F_{(2,345)}=2.38 ; p=0.1106\right)$. Although a four-way interaction is difficult to interpret, Figure $3, D$ and $E$ (graphs on right), illustrates that delay period activity during the DMS task is greater than delay period activity during the $\mathrm{CON}$ task for the $\mathrm{NO}$
$-34, y=-12, z=-26 ; Z=2.10 ; p_{\text {uncor }}=0.018$; statistical trend) (Fig. $4 A$, brain image on right), and in the right hippocampal body (peak at $x=34, y=-26, z=-14 ; Z=2.82$; 
$\left.p_{\text {uncor }}=0.001\right)($ Fig. $5 A$, brain image on left) and left hippocampal body (peak at $x=-30, y=-30, z=-12 ; Z=2.45$; $p_{\text {uncor }}=0.007$; statistical trend) (Fig. $5 A$, brain image on left). When the same analysis was done on the SCOP data, activity in these regions was not present (Figs. $4 B$, $5 B)$. There was only a small and statistically nonsignificant activation in the right mid-FG (peak at $x=30, y=-32, z=$ $-22 ; Z=2.34 ; p_{\text {uncor }}=0.01$; statistical trend; data not shown). Results from a two-sample $t$ test comparing the NO DRUG group with the SCOP group was consistent with this finding: the NO DRUG group had a significantly greater activation in the right hippocampal body (peak at $x=34, y=-26, z=-14 ; Z=$ 3.02; $p_{\text {uncor }}=0.001$ ) (Fig. $5 C$, brain image on left), in the left hippocampal head (peak at $x=-28, y=-14, z=-10 ; Z=$ $2.47 ; p_{\text {uncor }}=0.007$; statistical trend) (Fig. $5 C$, brain image on left), and in the right mid-FG (peak at $x=26, y=-50, z=$ $\left.-16 ; Z=3.10 ; p_{\text {uncor }}=0.001\right)$. There was also a greater activation in the right posterior PHG for the NO DRUG group compared with the SCOP group. However, the latter activation difference was small and statistically not significant. It extended from the mid-FG cluster to the posterior PHG (peak at $x=22, y=-30, z=-10$; $Z=2.33 ; p_{\text {uncor }}=0.010$; statistical trend; data not shown). There was no statistically significant activation difference in the right or left PRC/ERC for this comparison.

We repeated the same analysis collapsed across match and nonmatch trials. This additional analysis revealed greater activation in the right posterior $\mathrm{PHG}$ (peak at $x=26, y=-30, z=-14 ; Z=3.04 ; p_{\text {uncor }}=0.001$ ) (Fig. $4 C$, brain image on left) and also in the right PRC/ERC (peak at $x=40, y=-12, z=-26 ; Z=3.23 ; p_{\text {uncor }}=0.001$ ) (Fig. $4 C$, brain image on right) and left PRC/ERC (peak at $x=-36$, $y=-16, z=-30 ; Z=3.05 ; p_{\text {uncor }}=0.001$ ) (Fig. $4 C$, brain image on right) in NO DRUG subjects compared with SCOP subjects, possibly indicating that encoding-related delay activity in these regions does not distinguish between stimuli that were encountered once (nonmatch trials) and stimuli that were encountered twice (match trials) before the post-scan SMT. Conversely, this analysis did not show activation in the hippocampus. Activity in the hippocampus was only present when sample stimuli were seen only once before the post-scan SMT. This may indicate that the hippocampus plays a different role than the PRC/ERC in encoding (for review, see Squire et al., 2004). Interestingly, the SCOP group showed greater encoding-related memory delay activation in the left hippocampal head (peak at $x=-24, y=-8, z=-22$; $Z=3.18 ; p_{\text {uncor }}=0.001$ ) and a statistical trend for a significant activation difference in the right hippocampal head/uncus (peak at $x=20, y=-4, z=-24 ; Z=2.38 ; p_{\text {uncor }}=0.009$ ) than the NO DRUG group for this comparison (across match and nonmatch trials; data not shown).

\section{Hipp. Body Hipp.Body Nonmatch Match NO DRUG}

D
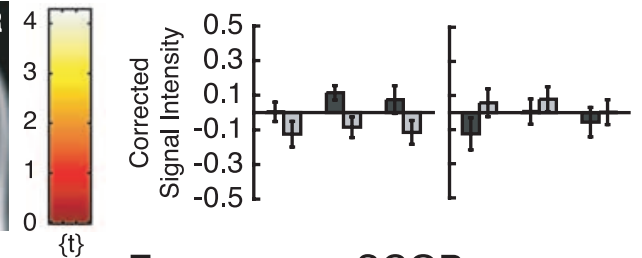

E
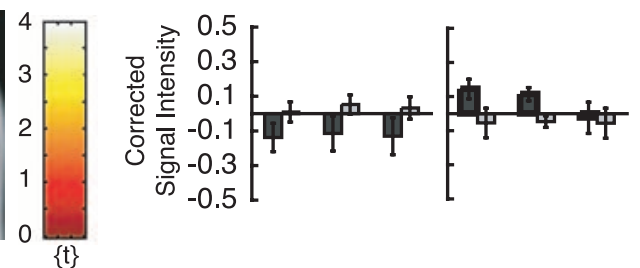

SMT5

SMT1-4

Figure 5. fMRI activation from long-term encoding analysis. Only activation within ROIs is superimposed on canonical average T1-weighted ICBM/MNI brain. $A$, NO DRUG group, hippocampal body activation, bilateral, $y=-26$, for nonmatch trials (stimul once), depicted on the left, and absence of hippocampal head activation, $y=-2$, for match trials (stimuli seen twice), nonmatch trials (stimuli seen once), depicted on the left, and presence of hippocampal head activation, $y=-2$, for match trials presentation (T) from the right hippocampal body for nonmatch trials (stimuli seen once), in the NO DRUG group, depicted on the eft, and for the left hippocampal body for match trials (stimuli seen twice), in the NO DRUG group, depicted on the right. $\boldsymbol{E}$, Same as $\boldsymbol{D}$ but for the SCOP group. $y$-Axes, Signal intensity grand mean scaled to 100 and global calculation using mean voxel value (within per image full mean/8 mask). R, Right hemisphere; L, left hemisphere.

When this analysis was repeated separately for match and nonmatch trials for the SCOP group, it became evident that this differential activity in the left hippocampal head (peak at $x=$ $-18, y=-6, z=-28 ; Z=2.00$; not significant; $p_{\text {uncor }}=0.023$ ) and in the right hippocampal head (peak at $x=20, y=-2, z=$ $-24 ; Z=3.11 ; p_{\text {uncor }}=0.001$ ) (Fig. $5 B$, brain image on right) in the SCOP group was present only for match trials, when stimuli were seen twice before SMT performance, but not for nonmatch trials, when stimuli were seen once before SMT performance. Only the activation in the right hippocampal head was statistically significant for this analysis. A two-sample $t$ test comparing the two groups directly confirmed this finding. There was a statistical trend toward activation in the left hippocampal head (peak at $x=-26, y=-8, z=-20 ; Z=2.49 ; p_{\text {uncor }}=0.006$; statistical trend) and in the right hippocampal head/uncus (peak at $x=20, y=-4, z=-24 ; Z=2.24 ; p_{\text {uncor }}=0.012$; statistical trend) (Fig. $5 B, C$, brain images on right) for match trials (SCOP $>$ NO DRUG), when stimuli were encountered twice before post-scan SMT performance. This activation pattern was not present in the NO DRUG group (Fig. $5 A$, brain images on right).

Repeated-measures ANOVAs on extracted signal-intensity 
Table 2. Repeated-measures ANOVA: subsequent memory analysis, delay period activity (nonmatch trials)

\begin{tabular}{|c|c|c|c|c|c|c|}
\hline \multirow[b]{2}{*}{ Source } & \multicolumn{6}{|l|}{$F$} \\
\hline & $\mathrm{df}$ & $\begin{array}{l}\mathrm{RPRC/ERC} \\
(34,-10,-28)^{a}\end{array}$ & $\begin{array}{l}\mathrm{LPRC/ERC} \\
(-34,-12,-26)^{a}\end{array}$ & $\begin{array}{l}\text { R Hipp. Body } \\
(34,-26,-14)^{a}\end{array}$ & $\begin{array}{l}\text { L Hipp. Body } \\
(-30,-30,-12)^{a}\end{array}$ & $\begin{array}{l}\text { R PHG/mid-FG } \\
(24,-24,-24)^{a}\end{array}$ \\
\hline Group & 1 & $5.08^{*}$ & $3.44^{c}$ & 0.19 & 2.33 & 1.31 \\
\hline Trial $^{b}$ & 1 & $3.64^{c}$ & $5.03^{*}$ & $7.80^{* *}$ & 0.00 & 0.55 \\
\hline Memory & 1 & $4.76^{*}$ & $6.35^{*}$ & $3.12^{c}$ & $7.85^{* *}$ & $5.50 *$ \\
\hline Event & 2 & 0.25 & 0.21 & 0.06 & $2.33^{c}$ & 1.60 \\
\hline Group $\times$ trial & 1 & 1.61 & 0.00 & 0.04 & $4.66^{*}$ & $3.50^{c}$ \\
\hline Group $\times$ memory & 1 & 1.28 & $4.44^{*}$ & $9.9^{* *}$ & 2.65 & 1.79 \\
\hline Group $\times$ event & 2 & 0.01 & 0.26 & 0.48 & 0.66 & 0.21 \\
\hline Trial $\times$ memory & 1 & 0.44 & 0.85 & $3.70^{c}$ & $5.54^{*}$ & $3.04^{c}$ \\
\hline Trial $\times$ event & 2 & 0.04 & 0.10 & 0.26 & 0.10 & 0.15 \\
\hline Memory $\times$ event & 2 & 0.01 & 0.02 & 0.22 & 0.11 & 0.49 \\
\hline Group $\times$ trial $\times$ memory & 1 & $4.75^{*}$ & 2.33 & 0.74 & $5.39 *$ & $4.71^{*}$ \\
\hline Group $\times$ trial $\times$ event & 2 & 0.22 & 0.06 & 0.35 & 0.73 & 0.14 \\
\hline Group $\times$ memory $\times$ event & 2 & 0.11 & 0.09 & 0.92 & $2.78^{c}$ & 0.21 \\
\hline Trial $\times$ memory $\times$ event & 2 & 0.04 & 0.04 & 0.22 & 0.06 & 0.10 \\
\hline Group $\times$ trial $\times$ memory $\times$ event & 2 & 0.08 & 0.14 & 0.39 & 0.13 & 0.05 \\
\hline Errors & 324 & $(0.24)$ & $(0.24)$ & $(0.15)$ & $(0.08)$ & $(0.60)$ \\
\hline
\end{tabular}

Note that values enclosed in parentheses represent mean square errors. R, Right; L, left; Hipp., hippocampal. $p<0.05$; ${ }^{* *} p<0.01$.

aPeak Talairach coordinates $(x, y, z)$ in MNI space.

${ }^{b}$ DMS trial (match vs nonmatch).

'Statistical trend $(p \leq 0.1)$.

Table 3. Repeated-measures ANOVA: subsequent memory analysis, delay period activity (all trials)

\begin{tabular}{|c|c|c|c|c|}
\hline \multirow[b]{2}{*}{ Source } & \multicolumn{4}{|l|}{$F$} \\
\hline & df & $\begin{array}{l}\text { R PHG/mid-FG } \\
(26,-32,-14)^{a}\end{array}$ & $\begin{array}{l}\mathrm{RPRC} / \mathrm{ERC} \\
(38,-12,-26)^{a}\end{array}$ & $\begin{array}{l}\mathrm{LPRC} / \mathrm{ERC} \\
(-36,-16,-30)^{a}\end{array}$ \\
\hline Group & 1 & 0.08 & 1.93 & 2.30 \\
\hline Trial $^{b}$ & 1 & 2.07 & $5.12^{*}$ & $3.29^{c}$ \\
\hline Memory & 1 & $12.18^{* * *}$ & $4.13^{*}$ & $3.39^{c}$ \\
\hline Event & 2 & $24.16^{* * *}$ & 0.57 & 0.03 \\
\hline Group $\times$ trial & 1 & 0.04 & 0.74 & 0.19 \\
\hline Group $\times$ memory & 1 & $13.9^{* * *}$ & $2.82^{c}$ & $4.78^{*}$ \\
\hline Group $\times$ event & 2 & 0.84 & 0.04 & 0.08 \\
\hline Trial $\times$ memory & 1 & 0.01 & 0.28 & 0.51 \\
\hline Trial $\times$ event & 2 & 0.11 & 0.2 & 0.11 \\
\hline Memory $\times$ event & 2 & 0.41 & 0.11 & 0.02 \\
\hline Group $\times$ trial $\times$ memory & 1 & 1.60 & $3.57^{c}$ & $5.28^{*}$ \\
\hline Group $\times$ trial $\times$ event & 2 & 0.03 & 0.06 & 0.08 \\
\hline Group $\times$ memory $\times$ event & 2 & 1.04 & 0.45 & 0.13 \\
\hline Trial $\times$ memory $\times$ event & 2 & 0.20 & 0.01 & 0.06 \\
\hline Group $\times$ trial $\times$ memory $\times$ event & 2 & 0.11 & 0.07 & 0.09 \\
\hline Errors & 324 & $(0.14)$ & $(0.15)$ & $(0.25)$ \\
\hline
\end{tabular}

Note that values enclosed in parentheses represent mean square errors. R, Right; L, left. ${ }^{*} p<0.05 ;{ }^{* * *} p<0.001$.

aPeak Talairach coordinates $(x, y, z)$ in MNI space.

${ }^{b}$ DMS trial (match vs nonmatch).

'Statistical trend $(p \leq 0.1)$.

time series from these activation peaks (derived from the NO DRUG group, analysis restricted to nonmatch trials) tested for the effects of group (NO DRUG vs SCOP), DMS trial (match vs nonmatch trials), memory (SMT5 vs SMT1-4), and event (SAMPLE vs DELAY vs TEST), as well as for interactions of these terms. Because we were interested in whether subsequent memory (SMT5 vs SMT1-4) was modulated by scopolamine, we were mainly interested in finding main effects and/or interactions involving the factors memory (SMT5 vs SMT1-4) and group (NO DRUG vs SCOP). Please note that data from repeated-measures ANOVAs based on coordinates extracted from a composite statistical parametric map from a one-sample $t$ test on all subjects (NO DRUG and SCOP groups) are available as supplemental data (available at www.jneurosci.org as supplemental material). This ANOVA allowed us to investigate whether there was greater sustained delay activity for items that were remembered with high confidence on the post-scan SMT (SMT5) than for those that were not (SMT1-4), and it allowed us to investigate whether this sustained encoding signal during the delay was attenuated by scopolamine. Group $\times$ memory and group $X$ trial $\times$ memory interactions provide answers to these questions. Group $X$ memory interactions show differences between the two groups as a function of subsequent memory (SMT5 vs SMT1-4), and group $\times$ trial $\times$ memory interactions reveal differences between groups as a function of both subsequent memory and the number of stimulus presentations before SMT performance (one presentation, nonmatch trials; two presentations, match trials). In addition, main effects of group, DMS trial, and memory are reported. Results of all repeated-measures ANOVAs corresponding to the coordinates extracted from the subsequent memory analysis restricted to nonmatch trials are summarized in Table 2, and those extracted from the subsequent memory analysis on all trials are summarized in Table 3.

The ANOVA on the time series from the right PRC/ERC revealed a significant main effect of group $\left(F_{(1,324)}=5.08 ; p=\right.$ $0.025)$, a significant main effect of memory $\left(F_{(1,324)}=4.76 ; p=\right.$ $0.030)$, and a significant group $\times$ DMS trial $\times$ memory interaction $\left(F_{(1,324)}=4.75 ; p=0.030\right)$, as well as a trend toward a main effect of DMS trial $\left(F_{(1,324)}=3.64 ; p=0.057\right)$. These results indicate that, in this anterior parahippocampal region, a subsequent memory effect (SMT5 > SMT1-4) was present throughout the DMS nonmatch trial in the NO DRUG group but not in the SCOP group (Fig. 4D, E, graphs on right, respectively).

In addition, the ANOVA on the time series from the left PRC/ ERC revealed a significant main effect of DMS trial $\left(F_{(1,324)}=\right.$ 
$5.03 ; p=0.026)$, a significant main effect of memory $\left(F_{(1,324)}=\right.$ $6.35 ; p=0.012)$, and a significant group $\times$ memory interaction $\left(F_{(1,324)}=4.44 ; p=0.036\right)$, as well as a trend toward a main effect of group $\left(F_{(1,324)}=3.44 ; p=0.064\right)$. Again, these results indicate a reduced subsequent memory effect throughout the DMS nonmatch trial in the SCOP group compared with the NO DRUG group (data not shown). In addition, focal linear tests clearly show that a subsequent memory effect (SMT5 $>$ SMT1-4) is present in nonmatch trial delays in the NO DRUG group $\left(F_{(1,324)}=5.43 ; p=0.020\right)$ but not in the SCOP group $\left(F_{(1,324)}=\right.$ $0.00 ; p=0.96)$.

The ANOVA on the time series from the right hippocampal body revealed a significant main effect of DMS trial $\left(F_{(1,324)}=\right.$ $7.80 ; p=0.006)$ and a significant group $\times$ memory interaction $\left(F_{(1,324)}=9.90 ; p=0.002\right)$. Furthermore, there was a trend toward a main effect of memory $\left(F_{(1,324)}=3.12 ; p=0.078\right)$ and toward a DMS trial $\times$ memory interaction $\left(F_{(1,324)}=3.70 ; p=\right.$ $0.055)$, again indicating a reduced subsequent memory effect throughout the DMS nonmatch trial in the SCOP group compared with the NO DRUG group (Fig. 5D, E, graphs on left).

The ANOVA on the time series from the left hippocampal body revealed a significant main effect of memory $\left(F_{(1,324)}=\right.$ $7.85 ; p=0.005)$ and significant group $\times$ DMS trial $\left(F_{(1,324)}=\right.$ 4.66; $p=0.032)$, DMS trial $\times$ memory $\left(F_{(1,324)}=5.54\right.$; $p=0.019)$, and group $\times$ DMS trial $\times$ memory interactions $\left(F_{(1,324)}=5.39 ; p=0.021\right)$. There was also a trend toward a group $\times$ memory $\times$ event interaction $\left(F_{(1,324)}=2.78 ; p=\right.$ $0.0864)$. The results on the time series from the left hippocampal body indicate that, not only was the subsequent memory effect reduced in the SCOP group compared with the NO DRUG group during the DMS nonmatch trial, the opposite trend was present for DMS match trials when sample stimuli were encountered twice before the post-scan SMT. For DMS match trials, the subsequent memory effect was increased in the SCOP group compared with the NO DRUG group (Fig. $5 E, D$, graphs on right). This finding is consistent with a greater subsequent memory effect during the DMS delay for SCOP subjects compared with NO DRUG subjects for stimuli encountered twice (Fig. 5, compare $B, C$, brain images on right).

And last, the ANOVA on the time series from the right posterior $\mathrm{PHG} /$ mid-FG revealed a significant main effect of memory $\left(F_{(1,324)}=5.50 ; p=0.020\right)$, a significant group $\times$ DMS trial $\times$ memory interaction $\left(F_{(1,324)}=4.71 ; p=0.031\right)$, and a trend toward a group $\times$ DMS trial interaction $\left(F_{(1,324)}=3.50 ; p=\right.$ $0.062)$. These results indicate that a subsequent memory effect (SMT5 > SMT1-4) was present throughout the DMS nonmatch trial in the NO DRUG group but not in the SCOP group (Fig. $4 D, E$, graphs on left, respectively).

In summary, statistically significant group $\times$ memory and group $\times$ DMS trial $\times$ memory interactions indicate that scopolamine reduces sustained activity during the DMS delay in the PRC/ERC, bilaterally, in the hippocampus, bilaterally, and in the right posterior PHG that predicted subsequent memory.

\section{Discussion}

This study demonstrates that muscarinic cholinergic blockade by scopolamine attenuates persistent activity in parahippocampal and hippocampal regions that is predictive of successful longterm encoding in healthy young individuals. In a separate analysis, scopolamine also reduces activity that is related to actively maintaining novel information in a short-term memory buffer, albeit only slightly. A region in the mid-FG/PHG, bilaterally, was recruited to actively maintain novel information in memory. Sus- tained delay activity bilaterally in the PRC/ERC, bilaterally in the hippocampus, and in the posterior PHG predicted subsequent long-term memory as assessed with the post-scan subsequent recognition memory test in healthy young individuals and therefore was related to long-term encoding. Although the effects of scopolamine on memory performance were relatively subtle, a scopolamine-challenge 90 min before encoding clearly reduced $\mathrm{fMRI}$ activity in these encoding-related brain regions.

The current findings support the hypothesis that persistent activity in parahippocampal neurons may enhance encoding, and that reduced muscarinic cholinergic receptor activation may reduce this activity and consequently may impair encoding. These results support computational models (Jensen and Lisman, 1996; Fransén et al., 2002; Hasselmo et al., 2002a,b; Koene et al., 2003) that propose that performance in both delayed matching tasks and long-term encoding could be enhanced by cholinergic activation of intrinsic mechanisms for sustained spiking activity as demonstrated in slice preparations of entorhinal cortex (Klink and Alonso, 1997; Egorov et al., 2002; Fransén et al., 2002).

\section{Relationship to behavioral studies}

These results are consistent with studies showing that scopolamine impairs encoding for long-term memory in both humans (Ghoneim and Mewaldt, 1977; Rosier et al., 1998; Sherman et al., 2003; Atri et al., 2004) and monkeys (Penetar and McDonough, 1983; Aigner and Mishkin, 1986; Aigner et al., 1991; Tang et al., 1997; Taffe et al., 1999). The changes in parahippocampal activation shown here are consistent with evidence that encoding is impaired by direct infusions of scopolamine into the perirhinal cortex, but not inferotemporal cortex or the dentate gyrus (Tang et al., 1997), and are consistent with monkey lesion studies that have established a role for the PRC in recognition memory (Meunier et al., 1993, 1996; Baxter and Murray, 2001).

Behavioral studies have shown that deficits are greater with larger doses of scopolamine and with longer retentions delays between encoding and recognition assessment (Bartus and Johnson, 1976; Penetar and McDonough, 1983; Aigner and Mishkin, 1986; Dunnett et al., 1990). This could explain the relatively mild behavioral deficit of the SCOP group on the post-scan subsequent recognition memory task, because our dose of scopolamine was relatively low and the delay between encoding and recognition assessment was short.

Consistent with our study, McGaughy et al. (2003) observed that cholinergic deafferentation of entorhinal cortex in rats caused deficits of performance in a delayed nonmatch-to-sample task with a 20 min delay when stimuli were novel but not when they were familiar. In the study presented here, we specifically focused on the effects of scopolamine on novel stimuli because previous neuroimaging studies demonstrated that activation of medial temporal cortices is strong for working memory with novel but not familiar stimuli (Ranganath and D'Esposito, 2001; Stern et al., 2001).

Behavioral studies can infer that it is long-term encoding and not retrieval or visual stimulus processing that is impaired under a scopolamine challenge. Here, we were able to directly link behavior with the sustained encoding signal by selectively averaging the fMRI activity during the DMS delay depending on whether or not the immediately preceding sample stimulus was remembered with high confidence on the behavioral recognition memory assessment. This study demonstrates that scopolamine reduces the fMRI signal in the MTL associated with long-term encoding of the stimuli. 


\section{Relationship to other neuroimaging studies}

These results are consistent with previous pharmacological neuroimaging studies that revealed decreased activation in the left fusiform gyrus during delayed recognition of abstract shapes (Rosier et al., 1999), decreased activation in both extent and magnitude of fusiform gyrus and hippocampus during face-name association learning (Sperling et al., 2002), and decreased activation during repetition priming in the left fusiform cortex (Thiel et al., 2002) after injection of scopolamine at encoding. In the present study, we used a subsequent memory paradigm that allowed us to investigate directly whether the fMRI response to novel stimuli was correlated with long-term encoding.

Furthermore, our design allowed us to examine whether scopolamine modulates activity during the delay period in a delayed matching task. Although previous studies have examined the role of acetylcholine in encoding activity that is dependent on the presence of the stimulus, this study and others reported from our laboratory (Schon et al., 2004) extend previous studies by analyzing encoding-related activity during the delay period in the absence of a stimulus. Our results demonstrate that encoding correlates with persistent activity during the delay period and supports the hypothesis that this persistent encoding signal may depend on acetylcholine levels. The persistent encoding signal is attenuated when acetylcholine receptor activation is reduced. Previous neuroimaging studies have examined the effects of enhanced acetylcholine levels using injections of the acetylcholinesterase inhibitor physostigmine in subjects performing visual (Furey et al., 2000a,b) or spatial (Bentley et al., 2004) working memory studies. These studies demonstrated that the fMRI signal specific to working memory encoding is enhanced in extrastriate visual regions, consistent with the reduction observed in the mid-FG with cholinergic blockade in our study. Our observed attenuation in this region with scopolamine, however, was small in magnitude. Furey et al. (2000a,b) and Bentley et al. (2004) attributed their findings to enhanced attention rather than enhanced working memory encoding. One possible reason why we observed active maintenance-related activity in the mid-FG/PHG may be because we used complex visual scenes as stimuli (Brewer et al., 1998; Kirchhoff et al., 2000).

Although it is possible that the reduction in sustained encoding during the DMS delay under scopolamine may be secondary to an attentional deficit, we argue against this, partly because of support from behavioral studies of encoding and modeling studies that link cellular mechanisms of sustained spiking to memory encoding. We do not rule out that attentional mechanisms are modulated by scopolamine, because it has been demonstrated that attention and working memory recruit similar brain networks that include prefrontal and posterior parietal regions (Awh and Jonides, 2001). However, hippocampal activity to novel stimuli has been demonstrated to be independent of whether they were attended to in an fMRI study using a bi-field visual-selective attention paradigm (Yamaguchi et al., 2004), providing additional evidence that the encoding-related hippocampal signal is not modulated by attention.

\section{Relationship to slice-recording data}

The hypothesis tested here was motivated by data from slice preparations showing that sustained spiking activity of parahippocampal neurons depends on activation of muscarinic acetylcholine receptors, which activate a calcium-dependent cation current (Klink and Alonso, 1997; Egorov et al., 2002). This provides an intrinsic mechanism for sustained spiking activity that does not depend on previous strengthening of synapses. Compu- tational modeling shows that the persistent spiking in these neurons for relatively prolonged periods of time may be an ideal mechanism for sustaining information about novel stimuli in a short-term memory buffer (Lisman and Idiart, 1995; Fransén et al., 2002). Additional modeling studies demonstrate that such a memory buffer can enhance encoding into long-term memory (Jensen and Lisman, 1996; Hasselmo et al., 2002a,b; Koene et al., 2003). These models suggested the hypothesis, confirmed here, that scopolamine should reduce delay activity related to encoding for subsequent memory. These models also suggested that loss of cholinergic modulation should impair memory for novel but not familiar stimuli, as demonstrated in another recent study (McGaughy et al., 2003).

Data from slices suggests that activation may be resistant to distracters. During sustained spiking activity of single entorhinal neurons, hyperpolarizing pulses that are several seconds in length shut off the spiking activity but do not prevent sustained spiking from starting again at the end of the hyperpolarization (Klink and Alonso, 1997). Thus, distracter stimuli during the delay period might temporarily reduce activation but should not prevent a subsequent memory effect associated with the delay period.

We observed a dissociation between brain areas related to active maintenance (mid-FG/PHG) and those related to longterm encoding (PRC/ERC, hippocampus, and posterior PHG) during the delay period of a delayed matching task. This finding demonstrates that it is not the concepts of working memory and long-term memory that are dissociable but underlying processes, in this case "active maintenance" and "long-term encoding." Our data suggest that both processes can be active simultaneously during working memory task performance.

Consistent with predictions from computational modeling studies, we conclude that the long-term encoding-related persistent activity during brief memory delays in the parahippocampal gyrus and in the hippocampus can be modulated by acetylcholine at muscarinic receptors in humans. This sustained encodingrelated delay activity is reduced when muscarinic cholinergic receptor activation is reduced. Furthermore, our data indicate that the fMRI signal is more sensitive to cholinergic modulation than behavior. This has implications for the use of fMRI as a biomarker for early detection and treatment response of Alzheimer's disease and other acetylcholine-deficit dementias.

\section{References}

Aigner TG, Mishkin M (1986) The effects of physostigmine and scopolamine on recognition memory in monkeys. Behav Neural Biol 45:81-87.

Aigner TG, Walker DL, Mishkin M (1991) Comparison of the effects of scopolamine administered before and after acquisition in a test of visual recognition memory in monkeys. Behav Neural Biol 55:61-67.

Atri A, Sherman S, Norman KA, Kirchhoff BA, Nicolas MM, Greicius MD, Cramer SC, Breiter HC, Hasselmo ME, Stern CE (2004) Blockade of central cholinergic receptors impairs new learning and increases proactive interference in a word paired-associate memory task. Behav Neurosci 118:223-236.

Awh E, Jonides J (2001) Overlapping mechanisms of attention and spatial working memory. Trends Cogn Sci 5:119-126.

Bartus RT, Johnson HR (1976) Short-term memory in the rhesus monkey: disruption from the anti-cholinergic scopolamine. Pharmacol Biochem Behav 5:39-46.

Baxter MG, Murray EA (2001) Opposite relationship of hippocampal and rhinal cortex damage to delayed nonmatching-to-sample deficits in monkeys. Hippocampus 11:61-71.

Bentley P, Husain M, Dolan RJ (2004) Effects of cholinergic enhancement on visual stimulation, spatial attention, and spatial working memory. Neuron 41:969-982.

Boynton GM, Engel SA, Glover GH, Heeger DJ (1996) Linear systems anal- 
ysis of functional magnetic resonance imaging in human V1. J Neurosci 16:4207-4221.

Brewer JB, Zhao Z, Desmond JE, Glover GH, Gabrieli JD (1998) Making memories: brain activity that predicts how well visual experience will be remembered. Science 281:1185-1187.

Buckner RL (1998) Event-related fMRI and the hemodynamic response. Hum Brain Mapp 6:373-377.

Buckner RL, Snyder AZ, Sanders AL, Raichle ME, Morris JC (2000) Functional brain imaging of young, nondemented, and demented older adults. J Cogn Neurosci 12 [Suppl 2]:24-34.

Cohen JD, MacWhinney B, Flatt M, Provost J (1993) PsyScope: a new graphic interactive environment for designing psychology experiments. Behav Res Methods Instr Comp 25:257-271.

Courtney SM, Ungerleider LG, Keil K, Haxby JV (1997) Transient and sustained activity in a distributed neural system for human working memory. Nature 386:608-611.

Courtney SM, Petit L, Maisog JM, Ungerleider LG, Haxby JV (1998) An area specialized for spatial working memory in human frontal cortex. Science 279:1347-1351.

Dale AM, Buckner RL (1997) Selective averaging of rapidly presented individual trials using fMRI. Hum Brain Mapp 5:329-340.

Davachi L, Wagner AD (2002) Hippocampal contributions to episodic encoding: insights from relational and item-based learning. J Neurophysiol 88:982-990.

Davachi L, Mitchell JP, Wagner AD (2003) Multiple routes to memory: distinct medial temporal lobe processes build item and source memories. Proc Natl Acad Sci USA 100:2157-2162.

Dunnett SB, Wareham AT, Torres EM (1990) Cholinergic blockade in prefrontal cortex and hippocampus disrupts short-term memory in rats. NeuroReport 1:61-64.

Egorov AV, Hamam BN, Fransén E, Hasselmo ME, Alonso AA (2002) Graded persistent activity in entorhinal cortex neurons. Nature 420:173-178.

Elliott R, Dolan RJ (1999) Differential neural responses during performance of matching and nonmatching to sample tasks at two delay intervals. J Neurosci 19:5066-5073.

Fernàndez G, Weyerts H, Schrader-Bolsche M, Tendolkar I, Smid HG, Tempelmann C, Hinrichs H, Scheich H, Elger CE, Mangun GR, Heinze HJ (1998) Successful verbal encoding into episodic memory engages the posterior hippocampus: a parametrically analyzed functional magnetic resonance imaging study. J Neurosci 18:1841-1847.

Fernàndez G, Brewer JB, Zhao Z, Glover GH, Gabrieli JD (1999) Level of sustained entorhinal activity at study correlates with subsequent cuedrecall performance: a functional magnetic resonance imaging study with high acquisition rate. Hippocampus 9:35-44.

Fransén E, Alonso AA, Hasselmo ME (2002) Simulations of the role of the muscarinic-activated calcium-sensitive nonspecific cation current INCM in entorhinal neuronal activity during delayed matching tasks. J Neurosci 22:1081-1097.

Furey ML, Pietrini P, Haxby JV (2000a) Cholinergic enhancement and increased selectivity of perceptual processing during working memory. Science 290:2315-2319.

Furey ML, Pietrini P, Alexander GE, Schapiro MB, Horwitz B (2000b) Cholinergic enhancement improves performance on working memory by modulating the functional activity in distinct brain regions: a positron emission tomography regional cerebral blood flow study in healthy humans. Brain Res Bull 51:213-218.

Genovese CR, Lazar NA, Nichols T (2002) Thresholding of statistical maps in functional neuroimaging using the false discovery rate. NeuroImage 15:870-878.

Ghoneim MM, Mewaldt SP (1975) Effects of diazepam and scopolamine on storage, retrieval and organizational processes in memory. Psychopharmacologia 44:257-262.

Ghoneim MM, Mewaldt SP (1977) Studies on human memory: the interactions of diazepam, scopolamine, and physostigmine. Psychopharmacology (Berl) 52:1-6.

Hasselmo ME, Hay J, Ilyn M, Gorchetchnikov A (2002a) Neuromodulation, theta rhythm and rat spatial navigation. Neural Netw 15:689-707.

Hasselmo ME, Cannon RC, Koene RA (2002b) A simulation of parahippocampal and hippocampal structures guiding spatial navigation of a virtual rat in a virtual environment: a functional framework for theta theory. In: The parahippocampal region: organization and role in cogni- tive functions (Witter MP, Wouterlood FG, eds), pp 139-161. Oxford: Oxford UP.

Jensen O, Lisman JE (1996) Novel lists of $7+/-2$ known items can be reliably stored in an oscillatory short-term memory network: interaction with long-term memory. Learn Mem 3:257-263.

Kirchhoff BA, Wagner AD, Maril A, Stern CE (2000) Prefrontal-temporal circuitry for episodic encoding and subsequent memory. J Neurosci 20:6173-6180.

Klink R, Alonso A (1997) Ionic mechanisms of muscarinic depolarization in entorhinal cortex layer II neurons. J Neurophysiol 77:1829-1843.

Koene RA, Gorchetchnikov A, Cannon RC, Hasselmo ME (2003) Modeling goal-directed spatial navigation in the rat based on physiological data from the hippocampal formation. Neural Netw 16:577-584.

Lisman JE, Idiart MA (1995) Storage of $7+/-2$ short-term memories in oscillatory subcycles. Science 267:1512-1515.

Maldjian JA, Laurienti PJ, Kraft RA, Burdette JH (2003) An automated method for neuroanatomic and cytoarchitectonic atlas-based interrogation of fMRI data sets. NeuroImage 19:1233-1239.

Mazziotta J, et al. (2001) A probabilistic atlas and reference system for the human brain: International Consortium for Brain Mapping (ICBM). Philos Trans R Soc Lond B Biol Sci 356:1293-1322.

McGaughy JA, Jindal M, Eichenbaum HB, Hasselmo ME (2003) Cholinergic deafferentiation of the entorhinal cortex in rats impairs encoding of novel but not familiar stimuli in delayed non-match to sample tasks (DNMS). Soc Neurosci Abstr 29:425.4.

Meunier M, Bachevalier J, Mishkin M, Murray EA (1993) Effects on visual recognition of combined and separate ablations of the entorhinal and perirhinal cortex in rhesus monkeys. J Neurosci 13:5418-5432.

Meunier M, Hadfield W, Bachevalier J, Murray EA (1996) Effects of rhinal cortex lesions combined with hippocampectomy on visual recognition memory in rhesus monkeys. J Neurophysiol 75:1190-1205.

Papke LE, Wooldridge JM (1996) Econometric methods for fractional response variables with an application to $401(\mathrm{k})$ plan participation rates. J Appl Econometrics 11:619-632.

Penetar DM, McDonough Jr JH (1983) Effects of cholinergic drugs on delayed match-to-sample performance of rhesus monkeys. Pharmacol Biochem Behav 19:963-967.

Postle BR, D’Esposito M (1999a) “What-Then-Where” in visual working memory: an event-related fMRI study. J Cogn Neurosci 11:585-597.

Postle BR, D'Esposito M (1999b) Dissociation of human caudate nucleus activity in spatial and nonspatial working memory: an event-related fMRI study. Brain Res Cogn Brain Res 8:107-115.

Postle BR, Zarahn E, D’Esposito M (2000) Using event-related fMRI to assess delay-period activity during performance of spatial and nonspatial working memory tasks. Brain Res Brain Res Protoc 5:57-66.

Ranganath C, D'Esposito M (2001) Medial temporal lobe activity associated with active maintenance of novel information. Neuron 31:865-873.

Rosier A, Cornette L, Orban GA (1998) Scopolamine-induced impairment of delayed recognition of abstract visual shapes. Neuropsychobiology 37:98-103.

Rosier AM, Cornette L, Dupont P, Bormans G, Mortelmans L, Orban GA (1999) Regional brain activity during shape recognition impaired by a scopolamine challenge to encoding. Eur J Neurosci 11:3701-3714.

Schon K, Hasselmo ME, LoPresti ML, Tricarico MD, Stern CE (2004) Persistence of parahippocampal representation in the absence of stimulus input enhances long-term encoding: a functional magnetic resonance imaging study of subsequent memory after a delayed match-to-sample task. J Neurosci 24:11088-11097.

Sherman SJ, Atri A, Hasselmo ME, Stern CE, Howard MW (2003) Scopolamine impairs human recognition memory: data and modeling. Behav Neurosci 117:526-539.

Sperling R, Greve D, Dale A, Killiany R, Holmes J, Rosas HD, Cocchiarella A, Firth P, Rosen B, Lake S, Lange N, Routledge C, Albert M (2002) Functional MRI detection of pharmacologically induced memory impairment. Proc Natl Acad Sci USA 99:455-460.

Squire LR, Stark CE, Clark RE (2004) The medial temporal lobe. Annu Rev Neurosci 27:279-306.

Stern CE, Sherman SJ, Kirchhoff BA, Hasselmo ME (2001) Hippocampal and prefrontal contributions to working memory tasks with novel and familiar stimuli. Hippocampus 11:337-346.

Suzuki WA, Miller EK, Desimone R (1997) Object and place memory in the macaque entorhinal cortex. J Neurophysiol 78:1062-1081. 
Taffe MA, Weed MR, Gold LH (1999) Scopolamine alters rhesus monkey performance on a novel neuropsychological test battery. Brain Res Cogn Brain Res 8:203-212.

Tang Y, Mishkin M, Aigner TG (1997) Effects of muscarinic blockade in perirhinal cortex during visual recognition. Proc Natl Acad Sci USA 94:12667-12669.

Thiel CM, Henson RN, Dolan RJ (2002) Scopolamine but not lorazepam modulates face repetition priming: a psychopharmacological fMRI study. Neuropsychopharmacology 27:282-292.

Wagner AD, Schacter DL, Rotte M, Koutstaal W, Maril A, Dale AM, Rosen BR, Buckner RL (1998) Building memories: remembering and forgetting of verbal experiences as predicted by brain activity. Science 281:1188-1191.
Worsley KJ, Marret S, Neelin P, Vandal AC, Friston KJ, Evans AC (1996) A unified statistical approach for determining significant signals in images of cerebral activation. Hum Brain Mapp 4:58-73.

Yamaguchi S, Hale LA, D’Esposito M, Knight RT (2004) Rapid prefrontal-hippocampal habituation to novel events. J Neurosci 24:5356-5363.

Yonelinas AP (1994) Receiver-operating characteristics in recognition memory: evidence for a dual-process model. J Exp Psychol Learn Mem Cogn 20:1341-1354.

Young BJ, Otto T, Fox GD, Eichenbaum H (1997) Memory representation within the parahippocampal region. J Neurosci 17:5183-5195.

Zarahn E, Aguirre G, D’Esposito M (1997) A trial-based experimental design for fMRI. NeuroImage 6:122-138. 